\title{
SPIN MATRIX EXPONENTIALS AND TRANSMISSION MATRICES*
}

\author{
BY \\ L. YOUNG \\ Stanford Research Institute
}

\begin{abstract}
The three Pauli spin matrices $\sigma_{i}(i=1,2,3)$ occur in the mechanical, especially quantum mechanical, theory of rotation in three-dimensional space. The three spin matrix exponentials are here defined as $\exp \left(\sigma_{i} x\right)$, where $x$ is the independent variable. Transmission matrices can be expressed in terms of spin matrix exponentials, thereby permitting a more systematic treatment of transmission line circuits.

Introduction. In the design of quarter-wave transformers, it has hitherto always been assumed that the guide wavelength is independent of position along the line. This is so, for instance, for $T E M$ modes; or for $T E_{\mathrm{O}_{\mathrm{n}}}$ modes in rectangular waveguide where the wide or ' $a$ ' dimension is kept constant. Such transformers, having guide wavelength independent of position, are called homogeneous transformers [1]. The first exact design formulas for ideal homogeneous quarter-wave transformers were given by Collin [2], who considered up to four sections. (The junction of two transmission lines when junction discontinuities are neglected, is called an "ideal transformer". This is analogous to two perfectly coupled coils of turns ratio $\left(Z_{2} / Z_{1}\right)^{1 / 2}$ and having infinite inductance.) The first complete synthesis procedure was given by Riblet [3]. The author later computed extensive numerical tables [4], which have been checked out experimentally on numerous occasions.

Riblet's synthesis procedure [3] is based on Richards' transformation [5] and Richards' theorem [6], and thereby depends on the commensurability of all transmission line sections in the circuit. The homogeneous quarter-wave transformer has also been used as a prototype circuit in the design of direct-coupled-cavity filters [7].

It has been shown that the performance of single-section quarter-wave transformers can always be improved by going from a homogeneous to an inhomogeneous design [8]. The analysis of inhomogeneous transformers of more than one section has only recently been undertaken [9], and the purpose of this paper is to present the mathematical tools which were developed for this purpose. A separate paper will deal with the design considerations and numerical results for multi-section inhomogeneous quarter-wave transformers [21].

Spin matrix exponentials. With line-lengths no longer commensurable, a more general formulation than is possible by Richards' transformation is required. For a systematic and compact treatment of transmission matrices, we shall employ the three Pauli spin matrices [10], which may be represented as follows:
\end{abstract}

${ }^{*}$ Received May 9, 1960. This paper was written while the author was at Westinghouse Electric Corporation; it is based on part of a dissertation for the degree of Doctor of Engineering at the Johns Hopkins University, Baltimore, Md., 1959. 


$$
\left.\begin{array}{l}
\sigma_{1}=\left\{\begin{array}{rr}
0 & 1 \\
1 & 0
\end{array}\right] \\
\sigma_{2}=j\left[\begin{array}{rr}
0 & -1 \\
1 & 0
\end{array}\right] \\
\sigma_{3}=\left[\begin{array}{rr}
1 & 0 \\
0 & -1
\end{array}\right]
\end{array}\right\} .
$$

They anti-commute among themselves, and their squares are equal to unity:

$$
\left.\begin{array}{c}
\sigma_{1} \sigma_{2}=-\sigma_{2} \sigma_{1}=j \sigma_{3} \\
\sigma_{2} \sigma_{3}=-\sigma_{3} \sigma_{2}=j \sigma_{1} \\
\sigma_{3} \sigma_{1}=-\sigma_{1} \sigma_{3}=j \sigma_{2}
\end{array}\right\}
$$

where $I$ is the unit matrix (idemfactor). We define the "spin matrix exponentials" by

$$
E_{i}(x)=\exp \left(x \sigma_{i}\right)=I \cosh x+\sigma_{i} \sinh x \quad(i=1,2,3)
$$

and their derivatives by

$$
\left.\begin{array}{rl}
E_{i}^{\prime}(x) & =\frac{d}{d x} E_{i}(x)=\sigma_{i} \exp \left(x \sigma_{i}\right) \\
& =\sigma_{i} E_{i}(x)=I \sinh x+\sigma_{i} \cosh x \quad(i=1,2,3)
\end{array}\right\} .
$$

They do not commute among themselves unless $i$ is the same, and then they commute and behave like ordinary exponentials:

$$
E_{i}(x) E_{i}(y)=E_{i}(x+y) .
$$

Also,

$$
E_{i}(x) \sigma_{i}=\sigma_{i} E_{i}(x)
$$

but

$$
E_{i}(x) \sigma_{i}=\sigma_{i} E_{i}(-x), \quad i \neq j .
$$

Similarly,

$$
\begin{aligned}
& E_{i}^{\prime}(x) E_{i}^{\prime}(y)=E_{i}^{\prime}(x+y), \\
& E_{i}^{\prime}(x) \sigma_{i}=\sigma_{i} E_{i}^{\prime}(x), \\
& E_{i}^{\prime}(x) \sigma_{i}=-\sigma_{i} E_{i}^{\prime}(-x), \quad i \neq j .
\end{aligned}
$$

Spin matrix exponentials occur in the mechanical (especially quantum mechanical) theory of rotation in three-dimensional space $[11,12]$. The matrix product

$$
Q=E_{3}\left(\frac{j \psi}{2}\right) E_{1}\left(\frac{j \theta}{2}\right) E_{3}\left(\frac{j \phi}{2}\right),
$$

where $\theta, \phi, \psi$ are the three Eulerian angles, yields a matrix whose four elements are the Cayley-Klein parameters [11]. At this point, we anticipate in order to complete the analogy: it will be seen later that Eq. (12) is like the transfer matrix of a single-section ideal transformer. This is not altogether surprising, since geometrical analogies have 
been developed before $[13,14,15]$, and projective charts have been used for the numerical solution of transmission line problems. The spinor theory of two-ports and its geometrical interpretation has also been discussed in general terms by Payne [16].

The Pauli spin matrices, together with the unit matrix, can be used to express any matrix [17] in the form of a quaternion, but the resulting more general form does not have the simplicity of the spin matrix exponentials which are well-suited for the analytic description and solution of inhomogeneous transformers.

The transfer matrix. There is no uniform terminology for the transformation matrices [18-20] which are used to analyze two-ports. The matrix defined by

$$
\left[\begin{array}{l}
a_{1} \\
b_{1}
\end{array}\right]=\mathbf{T}\left[\begin{array}{l}
a_{2} \\
b_{2}
\end{array}\right]
$$

where $a_{1}, b_{1}$ are the incident and reflected wave amplitudes at the input, and $a_{2}, b_{2}$ those at the output (Fig. 1), will be referred to as the transfer matrix. As only lossless two-ports are here considered, wave amplitudes may be defined in terms of power by

$$
\left.\begin{array}{c}
|a|^{2}=\begin{array}{l}
\text { power flow in the forward direction } \\
\text { (i.e. towards the load) }
\end{array} \\
|b|^{2}=\begin{array}{l}
\text { power flow in the backward direction } \\
\text { (i.e. towards the generator) }
\end{array}
\end{array}\right\}
$$

The transmission coefficient, $T$, between two reference planes is the same (in both phase and amplitude) when going from left to right as when going from right to left. The transfer matrix can then be written [18]

$$
T=\left[\begin{array}{cc}
\frac{1}{T} & -\frac{\Gamma_{2}}{T} \\
\frac{\Gamma_{1}}{T} & T-\frac{\Gamma_{1} \Gamma_{2}}{T}
\end{array}\right],
$$

where $T$ is the (unique) transmission coefficient, $\Gamma_{1}$ is the reflection coefficient seen at the input (on the left) when a matched load is placed at the output (on the right), and $\Gamma_{2}$ is similarly defined for the output side.

We may, by appropriate choice of reference planes, let

$$
\Gamma_{1}=-\Gamma_{2}=\Gamma \quad \text { (say). }
$$

From energy considerations:

1. Let $b_{2}=0$ in Fig. 1 . Then

$$
|\mathrm{I}|^{2}+|T|^{2}=1 \text {. }
$$

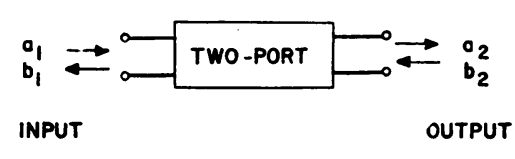

$$
\left(\begin{array}{l}
a_{1} \\
b_{1}
\end{array}\right)=T\left(\begin{array}{l}
a_{2} \\
b_{2}
\end{array}\right)
$$

FIG. 1. Defining the transfer matrix. 
2. Let $a_{2}=0$ in Fig. 1. Then

$$
\left|T^{2}+\Gamma^{2}\right|=1 \text {. }
$$

Comparing Eqs. (17) and (18), we infer that, under the condition (16), the transmission coefficient vector is parallel to the reflection coefficient vector [22]:

$$
T \text { parallel to } \Gamma \text {. }
$$

Now further choose the reference planes so that

$$
\Gamma=\text { real. }
$$

The transfer matrix then reduces to:

$$
\mathrm{T}=\frac{1}{T}\left(\begin{array}{ll}
1 & \Gamma \\
\Gamma & 1
\end{array}\right)
$$

The diagonal and anti-diagonal parts of T. From Eq. (15) it follows that for a reflectionless two-port, $\mathbf{T}$ must be a diagonal matrix. Let

$$
\mathrm{T}=\left[\begin{array}{ll}
T_{11} & T_{12} \\
T_{21} & T_{22}
\end{array}\right]
$$

Two useful concepts are

$$
\begin{aligned}
\operatorname{Di}(\mathbf{T}) & =\left[\begin{array}{ll}
T_{11} & 0 \\
0 & T_{22}
\end{array}\right) \\
& =\text { diagonal part of } \mathbf{T} \\
\operatorname{Ag}(\mathbf{T}) & =\left[\begin{array}{ll}
0 & T_{12} \\
T_{21} & 0
\end{array}\right) \\
& =\text { anti-diagonal part of } \mathbf{T} .
\end{aligned}
$$

For zero reflection,

$$
\operatorname{Ag}(\mathbf{T})=\mathbf{0} .
$$

The ideal transformer. The magnitude of the reflection coefficient at an ideal transformer (Fig. 2) is

$$
|\Gamma|=\left|\frac{Z_{2}-Z_{1}}{Z_{2}+Z_{1}}\right| \text {. }
$$

Select both reference planes to be coincident in the plane of the junction itself, and choose $\Gamma$ to be real. This satisfies Eqs. (16) and (20) and therefore leads to the form (21) for the transfer matrix. $\Gamma$ is now determined except for sign. In agreement with Ref. [4], we (arbitrarily) pick the positive sign and let

$$
\Gamma=\frac{Z_{2}-Z_{1}}{Z_{2}+Z_{1}} .
$$




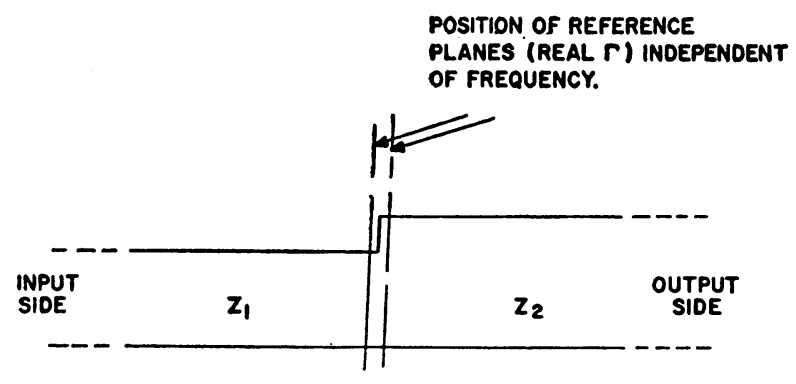

$z_{1}, z_{2}:$ CHARACTERISTIC IMPEDANCES.

Frg. 2. Ideal transformer.

Defining the "junction $V S W R$ " by

$$
V=\frac{Z_{2}}{Z_{1}}
$$

and the "log ratio" by

$$
\alpha=\frac{1}{2} \ln V
$$

the transfer matrix (21) of the ideal transformer reduces to

$$
\mathrm{T}=E_{1}(\alpha)
$$

which is the first spin matrix exponential as a function of the $\log$ ratio, $\alpha$, of the junction.

and since $\Gamma$ is real,

$$
\text { Also } \Gamma=\tanh \alpha \text {, }
$$

$$
T=\left(1-\Gamma^{2}\right)^{1 / 2}=\operatorname{sech} \alpha .
$$

Length of transmission line. A section of transmission line of electrical length $\theta$ radians (Fig. 3) has a transfer matrix

$$
\mathbf{T}=E_{3}(j \theta)
$$

which is the third spin matrix exponential of imaginary argument $j \theta$.

The ABCD matrix. The ABCD matrix of an ideal transformer is

$$
\mathrm{A}=E_{3}(\alpha)
$$

and that of a length of transmission line is

$$
\mathbf{A}=E_{1}(j \theta) .
$$

The transition between transfer and $A B C D$ matrices has thus been effected merely by interchanging suffixes of the first and third spin matrix exponentials.

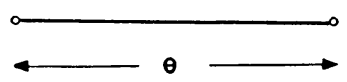

FIG. 3. Section of transmission line. 
Conclusion. The spin matrix exponentials exp $\left(\sigma_{i} x\right)$, where $x$ is either $j \theta(\theta=$ electrical line length) or the log ratio of a transformer, represent the transformation matrices of line sections and transformer steps. They are useful in the treatment of transmission line transformers, particularly inhomogeneous transformers, where the line lengths are incommensurable and Richards' transformation then does not apply [21].

Acknowledgment. The author owes much to the teaching and encouragement of Dr. W. H. Huggins of the Johns Hopkins University. The help of Dr. Ferdinand Hamburger, Jr. and Dr. C. F. Miller, also of the Johns Hopkins University, is gratefully acknowledged.

This work was made possible by the financial support of the Benjamin Garver Lamme Graduate Scholarship of the Westinghouse Electric Corporation for the academic year 1958-9.

\section{BiBLIOGRAPHY}

1. Leo Young, Concerning Riblet's theorem, Trans. IRE MTT-7, 477-478 (1959)

2. R. E. Collin, Theory and design of wide-band multisection quarter-wave transformers, Proc. IRE 43, 179-185 (1955)

3. H. J. Riblet, General synthesis of quarter-wave impedance transformers, Trans. IRE MTT-5, 36-43 (1957)

4. Leo Young, Tables for cascaded homogeneous quarter-wave transformers, Trans. IRE MTT-7, 233-237 (1959); Trans. IRE MTT-8, 243-244 (1960)

5. P. I. Richards, Resistor-transmission-line circuits, Proc. IRE 36, 217-220 (1948)

6. P. I. Richards, $A$ special class of functions with positive real part in a half-plane, Duke Math. J. 14, 777-786 (1947)

7. Leo Young, The quarter-wave transformer prototype circuit, Trans. IRE MTT-8, 483-489 (1960)

8. Leo Young, Optimum quarter-wave transformers Trans. IRE MTT-8, 478-482 (1960)

9. Leo Young, Design of microwave stepped transformers with applications to filters, Doctor of Engineering Dissertation, The Johns Hopkins University, Baltimore, Md., April 1959

10. See almost any book on Quantum Mechanics (e.g. P. A. M. Dirac, The principles of quantum mechanics, Oxford University Press, 3rd ed. p. 149)

11. H. Goldstein, Classical mechanics, Addison-Wesley Publishing Co., Reading, Mass., 1950, p.116

12. W. T. Payne, Elementary spinor theory, Am. J. Phys. 20, 253-262 (1952)

13. H. A. Wheeler, Wheeler monographs, vol. I, Wheeler Laboratories, Great Neck, New York 1953, Monograph No. 4, Geometric relations in circle diagrams of transmission-line impedance

14. G. A. Deschamps, New chart for the solution of transmission-line and polarization problems, Trans. IRE MTT-1, 5-13 (1953), or Electrical Communication 30, 247-254 (1953)

15. E. Folke Bolinder, Note on impedance transformations by the isometric circle method, Trans. IRE MTT-6, 111-112 (1958), where references to some of Bolinder's earlier papers are given

16. W. T. Payne, Spinor theory of four-terminal networks, J. Math. and Phys. 32, 19-33 (1953)

17. M. C. Pease, The analysis of broad-band microwave ladder networks, Proc. IRE 38, 180-183 (1950), Appendix

18. G. L. Ragan, Microwave transmission circuits, M. I. T. Rad. Lab. Ser., vol. 9, McGraw-Hill Book Co., New York 1948

19. E. F. Bolinder, Note on the matrix representation of linear two-port networks, Trans. IRE CT-4, 337-9 (1957)

20. Leo Young, Transformation matrices, Trans. IRE CT-5, 147-148 (1958)

21. Leo Young, Inhomogeneous quarter-wave transformers of two sections, Trans. IRE MTT, scheduled for Nov. 1960

22. The counterpart to Eq. (16) is $\Gamma_{1}=\Gamma_{2}=\Gamma$, which arises with symmetrical networks. In this case, the transmission coefficient and reflection coefficient vectors are orthogonal. See Leo Young, $A$ theorem on lossless symmetrical networks, Trans. IRE CT-7, 75 (1960) 